\title{
Atopic Dermatitis in Early Life: Evidence for at Least Three Phenotypes? Results from the GUSTO Study
}

\author{
Evelyn Xiu Ling Loo ${ }^{a}$ Lynette Pei-chi Shek ${ }^{a, d}$ Anne Goh ${ }^{f}$ Oon Hoe Teoh ${ }^{g}$ \\ Yiong Huak Chan ${ }^{\mathrm{e}}$ Shu E. Soh ${ }^{\mathrm{a}, \mathrm{c}}$ Seang Mei Saw ${ }^{\mathrm{c}}$ Kenneth Kwek ${ }^{\mathrm{h}}$ \\ Peter D. Gluckman ${ }^{i, j}$ Keith M. Godfrey ${ }^{k, I}$ Yap Seng Chong ${ }^{b}$ Bee Wah Lee ${ }^{a}$ \\ Hugo P. Van Bever ${ }^{\text {a, }}$.
}

\begin{abstract}
Departments of a Paediatrics and b Obstetrics and Gynaecology, Yong Loo Lin School of Medicine and 'Saw Swee Hock School of Public Health, National University of Singapore, ' Khoo Teck Puat-National University Children's Medical Institute, National University Hospital and 'Biostatistics Unit, Yong Loo Lin School of Medicine, National University

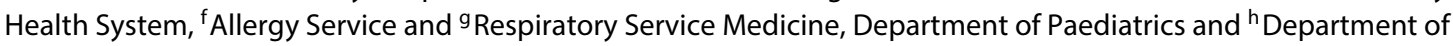
Maternal Fetal Medicine, KK Women's and Children's Hospital, and 'Growth, Development and Metabolism Programme, Singapore Institute for Clinical Sciences, Agency for Science, Technology and Research (A*STAR), Singapore, Singapore; jLiggins Institute, University of Auckland, Auckland, New Zealand; ${ }^{\mathrm{k}}$ NIHR Southampton Biomedical Research Centre, University of Southampton and University Hospital Southampton NHS Foundation Trust, and 'Medical Research Council Lifecourse Epidemiology Unit, Southampton, UK
\end{abstract}

\section{Key Words}

Atopic dermatitis · Phenotypes · Risk factors

\begin{abstract}
Background: Atopic dermatitis (AD) has been highlighted as a likely first step in the 'atopic march', emphasizing the need to define predisposing factors. Methods: We evaluated AD risk factors and phenotypes in an Asian mother-offspring cohort. We defined three phenotypes of doctor-diagnosed AD based on the time of onset of the disease: early AD occurring within the first 6 months of life, AD occurring between 6 and 12 months and late-onset AD starting after the age of 12 months. Results: Maternal allergic history was associated with an increased risk of developing early-onset AD (adjusted odds ratio (aOR) 20.46, 95\% confidence interval (CI) 2.73$153.15, p<0.01)$. Maternal allergic history and attendance at
\end{abstract}

a daycare centre increased the odds of the development of AD between 6 and 12 months (aOR 4.19, 95\% Cl 1.01-17.45, $p=0.049$ and aOR $11.42,95 \% \mathrm{Cl} 1.49-87.50, p=0.02$, respectively). Risk factors associated with increased odds of lateonset AD from 12 months were the consumption of probiotics between the age of 9 and 12 months and antibiotic treatment in the first 6 months of life (aOR 4.32,95\% Cl 1.07-17.45, $\mathrm{p}=0.04$ and aOR $3.11,95 \% \mathrm{Cl} 1.10-8.76, \mathrm{p}=0.03$, respectively). Early-onset AD was associated with an increased risk of developing allergic sensitization (aOR 46.51, 95\% Cl 3.44628.81 , p < 0.01). Conclusion: We found that early-onset AD was mainly associated with familial factors, while late-onset $A D$ was associated with the consumption of antibiotics or probiotics. The findings support the concept that different phenotypes of AD exist in young children.

(c) 2015 S. Karger AG, Basel

\section{KARGER 125}

(c) 2015 S. Karger AG, Base

$1018-2438 / 15 / 1664-0273 \$ 39.50 / 0$ 


\section{Introduction}

There is a wide geographical variation in the prevalence of allergic diseases such as atopic dermatitis (AD) in different regions [1], suggesting that there may be location-specific factors such as environment, lifestyle and microbial exposure affecting the onset of disease [2]. In Singapore, two International Study of Asthma and Allergies in Childhood surveys were conducted 7 years apart in 2 age groups (6-7 and 12-15 years). The prevalence of $\mathrm{AD}$, but not of allergic disorders, increased in both age groups over the 7 -year period [3]. AD is known to start early in life, with incidences of up to 45 and $60 \%$ having been reported in the first 6 and 12 months of life, respectively $[4,5]$. In a study of Singaporean school children, the reported prevalence of AD was $20.8 \%$ [6] while in another study, a chronic itchy rash was found to affect $22.1 \%$ of Singaporean children, although $\mathrm{AD}$ was only diagnosed in $34.4 \%$ of these cases $[6,7]$. AD has also been highlighted in many studies to be a likely first step of the 'atopic march'. Hence, it is vital to pinpoint the factors that may lead to or predispose a child to developing $\mathrm{AD}$ [8]. In this study, we evaluated risk factors and phenotypes of $\mathrm{AD}$ by studying its features in an Asian mother-offspring cohort, GUSTO (Growing Up in Singapore Towards healthy Outcomes). We postulated that different risk factors might be responsible for different $\mathrm{AD}$ phenotypes, according to the time of disease onset. We distinguished three phenotypes of $\mathrm{AD}$ based on the time of onset of the disease: early AD occurring within the first 6 months of life, AD occurring between 6 and 12 months and late-onset $\mathrm{AD}$ starting after the age of 12 months.

\section{Methods}

\section{Study Population and Questionnaires}

The methodology of the GUSTO study has been described [9, 10]. Briefly, we recruited healthy pregnant mothers who agreed to donate birth tissue (i.e. placenta, cord blood and cord) and consented to follow-up of their offspring. Mothers on chemotherapy, psychotropic drugs or with type I diabetes were excluded. Interviews were carried out and the information gathered included demographics, a family history of allergy, social data and lifestyle. During infancy, examinations of the babies were carried out at their homes at the age of 3 weeks and at 3, 6, 9, 12 and 15 months, following which, skin-prick testing (SPT) for common allergens was performed at the study clinic site, at the age of 18 months. In this study, the questionnaires included a case definition of $\mathrm{AD}$, adapted and modified from the International Study of Asthma and Allergy in Children, to ensure standardization and consistency during interviews and home visits [11]. Doctor-diagnosed AD cases were estimated on the basis of positive answers to the question: 'Has your child ever been diagnosed with eczema?' AD was classi- fied as YES when a subject had at least one YES answer at any visit and classified as NO when answers at all the visits were NO. A missing response was given if some of the answers from the visits were missing.

Socioeconomic status was assessed by asking respondents to state the total family income and the type of residence (public housing, condominium or landed houses). Environmental factors were assessed by asking respondents if the child was sent to a childcare/daycare centre, if pets such as cats and dogs were kept and if there was exposure to cigarette smoke. This study was approved by both the National Healthcare Group Domain Specific Review Board and the Singhealth Centralised Institutional Review Board.

SPT at the Age of 18 Months

At the 18-month clinic visit, children underwent SPT for aeroallergens (house-dust mites Dermatophagoides pteronyssinus, D. farinae and Blomia tropicalis) and food allergens (egg, peanut and cow's milk). A test was taken to be positive if the wheal size was at least $3 \mathrm{~mm}$, and if a histamine reaction was at least $3 \mathrm{~mm}$ and a buffer reaction $0 \mathrm{~mm}$.

\section{Statistical Analysis}

Statistical analysis was carried out using SPSS version 20.0 (IBM SPSS Statistics, Armonk, N.Y., USA). Logistic regression was used to determine the univariate associations between the potential risk factors and the outcome. Risk factors with a p value $<0.200$ in univariate analysis were then analyzed via multivariate logistic regression and the stepwise backward Wald method to determine if they were independent risk factors.

\section{Results}

\section{Demographic Characteristics of the Birth Cohort}

Of the 1,247 subjects recruited into the GUSTO cohort, 792 (63.5\%) subjects had responses (i.e. YES or NO) to questions related to doctor-diagnosed AD. Out of these 792 subjects, $51.9 \%$ were male and the majority were Chinese (60.2\%). Malays and Indians made up $24.2 \%$ and $15.5 \%$ of the study population, respectively.

\section{Prevalence Based on Questionnaires}

Doctor-diagnosed AD was reported by $23.6 \%$ (187/792). Of these, $53.5 \%(100 / 187)$ were diagnosed before the age of 6 months, $23.0 \%(43 / 187)$ had the diagnosis between 6 and 12 months and 25.1\% (47/187) were diagnosed after 12 months of age.

\section{Risk Factors of Doctor-Diagnosed AD}

A univariate analysis of risk factors for doctor-diagnosed $\mathrm{AD}$ is shown in table 1 . At the univariate level, risk factors associated with an increased risk of developing doctor-diagnosed AD were maternal consumption of alcohol, a maternal history of allergy, the maternal education level and the monthly household income (odds ratio (OR) 2.87, 
Table 1. Findings in bivariate analyses of doctor-diagnosed $\mathrm{AD}$ cases with explanatory and outcome parameters

\begin{tabular}{|c|c|c|c|}
\hline Outcome factor & Explanatory parameter & $\mathrm{p}$ value & OR (95\% CI) \\
\hline \multicolumn{4}{|c|}{ Doctor-diagnosed AD cases } \\
\hline & Has siblings & 0.06 & $0.69(0.47-1.02)$ \\
\hline & Maternal consumption of alcohol & 0.04 & $2.87(1.03-8.04)$ \\
\hline & Maternal smoking during pregnancy & 0.17 & $0.36(0.08-1.54)$ \\
\hline & Maternal allergic history & $<0.01$ & $2.27(1.42-3.62)$ \\
\hline & Paternal allergic history & 0.18 & $1.42(0.85-2.39)$ \\
\hline & Female gender & 0.02 & $0.68(0.49-0.94)$ \\
\hline & Maternal education level & 0.02 & $1.67(1.09-2.56)$ \\
\hline & Ethnicity (Chinese) & 0.03 & 1.00 \\
\hline & Ethnicity (Malay) & 0.38 & $0.84(0.57-1.24)$ \\
\hline & Ethnicity (Indian) & $<0.01$ & $0.48(0.28-0.83)$ \\
\hline & Birth weight & 0.12 & $1.00(1.000-1.001)$ \\
\hline & Birth length & 0.15 & $1.06(0.98-1.14)$ \\
\hline & Body fat & 0.17 & $2.32(0.70-7.72)$ \\
\hline & Monthly household income & $<0.01$ & $1.79(1.27-2.54)$ \\
\hline \multicolumn{4}{|c|}{ Doctor-diagnosed AD cases from birth to 6 months } \\
\hline & Maternal consumption of alcohol & 0.03 & $3.51(1.17-10.49)$ \\
\hline & Maternal allergic history & 0.01 & $2.07(1.16-3.72)$ \\
\hline & Paternal allergic history & 0.17 & $1.57(0.83-2.97)$ \\
\hline & Sibling allergic history & 0.17 & $2.36(0.70-7.94)$ \\
\hline & Maternal education level & 0.03 & $1.87(1.07-3.27)$ \\
\hline & Ethnicity (Chinese) & 0.16 & 1.00 \\
\hline & Ethnicity (Malay) & 0.78 & $0.93(0.57-1.53)$ \\
\hline & Ethnicity (Indian) & 0.06 & $0.49(0.24-1.02)$ \\
\hline & Monthly household income & 0.07 & $1.51(0.97-2.34)$ \\
\hline \multicolumn{4}{|c|}{ Doctor-diagnosed AD cases from 6 months to 12 months } \\
\hline & Maternal allergic history & 0.03 & $2.50(1.12-5.62)$ \\
\hline & Vaginal delivery & 0.11 & $0.60(0.32-1.13)$ \\
\hline & Antibiotic treatment in the first 6 months of life & 0.02 & $2.40(1.17-4.95)$ \\
\hline & Attended daycare & $<0.01$ & $3.56(1.38-9.21)$ \\
\hline & Having a cat & 0.11 & $2.85(0.80-10.13)$ \\
\hline & Having a dog & 0.09 & $2.37(0.87-6.47)$ \\
\hline & Living near an expressway & 0.17 & $0.36(0.08-1.53)$ \\
\hline & Female gender & $<0.01$ & $0.35(0.18-0.71)$ \\
\hline & Maternal education level & 0.03 & $0.41(0.18-0.92)$ \\
\hline \multicolumn{4}{|c|}{ Doctor-diagnosed AD cases from 12 to 18 months } \\
\hline & Has siblings & 0.10 & $0.58(0.30-1.12)$ \\
\hline & Prenatal exposure to smoke & 0.14 & $0.59(0.30-1.20)$ \\
\hline & Consumption of probiotics between 9 and 12 months & 0.06 & $2.85(0.95-8.61)$ \\
\hline & Antibiotic treatment in the first 6 months of life & 0.02 & $2.30(1.15-4.60)$ \\
\hline & Sum of skinfold thickness at birth & 0.20 & $1.09(0.96-1.24)$ \\
\hline & Maternal education level & 0.02 & $3.04(1.23-7.48)$ \\
\hline & Birth weight & 0.05 & $1.001(0.999-1.001)$ \\
\hline & Birth length & 0.07 & $1.13(0.99-1.30)$ \\
\hline & Monthly household income & $<0.01$ & $2.93(1.59-5.40)$ \\
\hline
\end{tabular}

95\% confidence interval (CI) 1.03-8.04, $\mathrm{p}=0.04$; OR 2.27, 95\% CI 1.42-3.62, $\mathrm{p} \leq 0.01$; OR 1.67, 95\% CI 1.09-2.56, $\mathrm{p}=$ 0.02 ; OR $1.79,95 \%$ CI 1.27-2.54, $\mathrm{p} \leq 0.01$, respectively). Factors associated with decreased odds of developing doctor-diagnosed $\mathrm{AD}$ in univariate analysis were female gender and Indian ethnicity (OR $0.68,95 \%$ CI $0.49-0.94, p=$ 0.02 and OR $0.48,95 \%$ CI $0.28-0.83, \mathrm{p} \leq 0.01$, respectively).
A multivariate analysis showed that having siblings and being of Indian ethnicity were associated with reduced odds of developing doctor-diagnosed AD (adjusted (a)OR 0.46, $95 \%$ CI $0.22-0.95, \mathrm{p}=0.04$ and $\mathrm{aOR} 0.14,95 \%$ CI 0.03-0.65, $\mathrm{p}=0.01$, respectively; table 2 ). A maternal allergic history was associated with increased odds of developing doctordiagnosed $\mathrm{AD}$ (aOR 3.15, 95\% CI 1.51-6.57, $\mathrm{p} \leq 0.01$ ). 
Table 2. Findings in the multivariate analyses of doctor-diagnosed AD cases with explanatory and outcome parameters

\begin{tabular}{|c|c|c|c|}
\hline Outcome factor & Explanatory parameter & $\mathrm{p}$ value & OR $(95 \% \mathrm{CI})$ \\
\hline \multicolumn{4}{|c|}{ Doctor-diagnosed AD cases } \\
\hline & Has siblings & 0.04 & $0.46(0.22-0.95)$ \\
\hline & Maternal consumption of alcohol & 0.28 & $3.27(0.39-27.53)$ \\
\hline & Prenatal smoking & 0.97 & $0.95(0.10-9.42)$ \\
\hline & Maternal allergic history & $<0.01$ & $3.15(1.51-6.57)$ \\
\hline & Paternal allergic history & 0.55 & $1.27(0.58-2.79)$ \\
\hline & Female gender & 0.24 & $2.01(0.63-6.38)$ \\
\hline & Maternal education level & 0.62 & $1.24(0.53-2.89)$ \\
\hline & Ethnicity (Chinese) & 0.04 & 1.00 \\
\hline & Ethnicity (Malay) & 0.85 & $0.92(0.41-2.11)$ \\
\hline & Ethnicity (Indian) & 0.01 & $0.14(0.03-0.65)$ \\
\hline & Birth weight & 0.29 & $1.00(0.998-1.01$ \\
\hline & Birth length & 0.68 & $0.95(0.75-1.20)$ \\
\hline & Body fat & 0.44 & $0.02(0.001-585.91)$ \\
\hline & Monthly household income & 0.73 & $0.85(0.34-2.11)$ \\
\hline \multicolumn{4}{|c|}{ Doctor-diagnosed AD cases from birth to 6 months } \\
\hline & Maternal allergic history & $<0.01$ & $20.46(2.73-153.15)$ \\
\hline & Paternal allergic history & 0.12 & $5.30(0.67-42.20)$ \\
\hline & Sibling allergic history & 0.32 & $2.50(0.41-15.19)$ \\
\hline & Maternal education level & 0.71 & $0.64(0.06-7.08)$ \\
\hline & Monthly household income & 0.48 & $2.61(0.18-37.96)$ \\
\hline \multicolumn{4}{|c|}{ Doctor-diagnosed AD cases from 6 to 12 months } \\
\hline & Maternal allergic history & 0.049 & $4.19(1.01-17.45)$ \\
\hline & Vaginal delivery & 0.72 & $0.75(0.15-3.61)$ \\
\hline & Antibiotic treatment in the first 6 months of life & 0.13 & $3.19(0.72-14.11)$ \\
\hline & Attended daycare & 0.02 & $11.42(1.49-87.50)$ \\
\hline & Having a dog & 0.16 & $3.76(0.60-23.59)$ \\
\hline & Female gender & 0.23 & $0.35(0.06-1.92)$ \\
\hline & Maternal education level & 0.04 & $0.16(0.03-0.88)$ \\
\hline \multicolumn{4}{|c|}{ Doctor-diagnosed AD cases from 12 to 18 months } \\
\hline & Has siblings & 0.54 & $0.75(0.30-1.87)$ \\
\hline & Prenatal exposure to smoke & 0.95 & $0.96(0.30-3.04)$ \\
\hline & Consumption of probiotics between 9 and 12 months & 0.04 & $4.32(1.07-17.45)$ \\
\hline & Antibiotic treatment in the first 6 months of life & 0.03 & $3.11(1.10-8.76)$ \\
\hline & Sum of skinfold thickness at birth & 0.27 & $1.14(0.90-1.44)$ \\
\hline & Maternal education level & 0.08 & $3.61(0.85-15.40)$ \\
\hline & Birth weight & 0.59 & $1.00(0.999-1.002)$ \\
\hline & Birth length & 0.78 & $0.96(0.70-1.30)$ \\
\hline & Monthly household income & 0.09 & $2.44(0.88-6.76)$ \\
\hline
\end{tabular}

The univariate analysis showed the risk factors associated with increased odds of developing early doctor-diagnosed $\mathrm{AD}$, i.e. before the age of 6 months, were maternal consumption of alcohol, a maternal allergic history and the maternal education level (OR 3.51, 95\% CI 1.17$10.49, \mathrm{p}=0.03$; OR 2.07, 95\% CI 1.16-3.72, $\mathrm{p}=0.01$; OR $1.87,95 \%$ CI 1.07-3.27, $\mathrm{p}=0.03$, respectively; table 1 ). In the multivariate analysis, a maternal allergic history remained a significant risk factor (aOR 20.46, 95\% CI 2.73$153.15, \mathrm{p} \leq 0.01$; table 2).

A maternal allergic history, antibiotic treatment in the first 6 months of life and attendance at a daycare centre were significantly associated with increased odds of developing $\mathrm{AD}$ between 6 and 12 months, while female gender and the maternal education level were protective effects at the univariate analysis level. The multivariate analysis revealed that a maternal allergic history and daycare centre attendance increased the odds of development of $\mathrm{AD}$ between the ages of 6 and 12 months (aOR 4.19, 95\% CI 1.01-17.45, $\mathrm{p}=0.049$ and aOR $11.42,95 \%$ CI 1.49-87.50, $\mathrm{p}=0.02$, respectively) while a higher level of maternal education reduced the odds (aOR 0.16, 95\% CI 0.03-0.88, $\mathrm{p}=0.04$; table 2).

The univariate analysis showed that antibiotic treatment in the first 6 months of life, the maternal education 
Table 3. Findings in bivariate and multivariate analyses of positive SPT at 18 months with explanatory and outcome parameters

\begin{tabular}{llrc}
\hline Outcome factor & Explanatory parameter & p value & OR (95\% CI) \\
\hline Bivariate analyses & & & \\
Positive SPT & Onset of AD by 6 months of age & $<0.01$ & $4.58(2.71-7.76)$ \\
& Maternal consumption of alcohol & 0.14 & $2.38(0.74-7.61)$ \\
& Paternal allergic history & 0.09 & $1.62(0.92-2.85)$ \\
& Sibling allergic history & 0.15 & $2.03(0.78-5.30)$ \\
& Breastfed till 3 months & 0.03 & $1.60(1.04-2.45)$ \\
& Breastfed till 6 months & 0.09 & $1.43(0.95-2.16)$ \\
& Breastfed till 18 months & 0.06 & $1.72(0.98-3.01)$ \\
& Antibiotic treatment in the first 6 months of life & 0.14 & $0.59(0.30-1.18)$ \\
& Having a cat & 0.12 & $2.29(0.81-6.50)$ \\
& Living near an expressway & 0.11 & $1.68(0.88-3.19)$ \\
& Exposed to air-conditioning & 0.14 & $0.65(0.37-1.15)$ \\
& Female gender & 0.10 & $0.72(0.48-1.07)$ \\
& Ethnicity (Chinese) & 0.04 & 1.00 \\
& Ethnicity (Malay) & 0.36 & $1.23(0.79-1.92)$ \\
& Ethnicity (Indian) & 0.04 & $0.50(0.26-0.97)$ \\
\hline Pultivariate analyses & & & \\
& Onset of AD by 6 months of age & $<0.01$ & $46.51(3.44-628.81)$ \\
& Sibling allergic history & 0.41 & $2.28(0.32-16.11)$ \\
& Antibiotic treatment in the first 6 months of life & 0.13 & $11.44(0.50-260.29)$ \\
& Living near an expressway & 0.44 & $2.37(0.27-21.24)$ \\
& Female gender & 0.22 & $3.37(0.49-23.24)$ \\
\hline
\end{tabular}

level and the monthly household income were associated with increased odds of late-onset $\mathrm{AD}$, i.e. between 12 and 18 months of age (OR 2.30, 95\% CI 1.15-4.60, $\mathrm{p}=0.02$; OR 3.04, 95\% CI 1.23-7.48, p = 0.02; OR 2.93, 95\% CI $1.59-5.40, \mathrm{p} \leq 0.01$, respectively). Multivariate analysis showed that consumption of probiotics between the ages of 9 and 12 months and antibiotic treatment in the first 6 months of life were associated with increased odds of developing $\mathrm{AD}$ between the ages of 12 and 18 months (aOR 4.32, 95\% CI 1.07-17.45, $\mathrm{p}=0.04$ and aOR 3.11, $95 \%$ CI 1.10-8.76, $\mathrm{p}=0.03$, respectively; table 2). Out of the 12 subjects who developed AD between the ages of 12 and 18 months and taken antibiotics in the first 6 months of life, the majority $(n=5)$ were due to respiratory tract infections.

\section{Risk Factors for Allergic Sensitization}

Of the 792 subjects who responded to the question on doctor-diagnosed AD, 696 underwent SPT for house-dust mites and food allergens. Sixty $(8.62 \%)$ had a positive SPT to house-dust mites while 18 (2.59\%) had a positive SPT to food allergens only. Six hundred and two subjects (86.5\%) did not have any positive SPTs while $16(2.3 \%)$ had positive SPTs to both dust mite and food allergens. In the multi- variate analyses, only an early onset of doctor-diagnosed AD by 6 months was associated with an increased risk of a positive SPT to house-dust mites and/or food allergens (aOR 46.51, 95\% CI 3.44-628.81, $\mathrm{p} \leq 0.01$; table 3).

\section{Discussion}

In this study, we defined three phenotypes of AD based on the time of onset of the disease: early $\mathrm{AD}$ occurring within the first 6 months of life, AD developing between the ages of 6 and 12 months and late-onset AD starting after the age of 12 months. In a previous study, there had been evidence of the presence of two variants of eczema that were attributed to different risk factors [12]. Therefore, because of the variations in the clinical presentation of $\mathrm{AD}$ (i.e. severity, skin type and age of onset), we postulated that different risk factors might be involved, mainly in the types of $\mathrm{AD}$ occurring at both ends of the spectrum (i.e. onset during the first months of life vs. onset beyond the age of 12 months).

The prevalence of doctor-diagnosed $\mathrm{AD}$ according to the questionnaire was $23.6 \%$, which was similar to that reported previously [7]. The involvement of parental his- 
tory of allergy in predisposing the infant to developing allergic diseases is well-established and widely reported by many groups [13-15]. Similar to our observations of Indian ethnicity reducing the odds of developing $\mathrm{AD}$ compared to being Chinese, another study conducted on the local population yielded similar findings of a higher frequency of $\mathrm{AD}$ in Chinese (21.6\%) compared to Indian children (16\%) [6]. The association of having siblings with a reduced odds of developing $\mathrm{AD}$ is consistent with the hygiene hypothesis, whereby an increased microbial load lowers the risk of developing allergic diseases.

Most studies on antibiotic exposure have focused on asthma as an end point $[16,17]$. As of yet, there is no conclusive evidence to show that antibiotic treatment is a risk factor for $\mathrm{AD}$. In a birth cohort study conducted in The Netherlands involving 2,764 families, in which information was collected on antibiotic exposure in the first 6 months of life, there was no association of antibiotic exposure with $\mathrm{AD}$ in the children who were followed up for 2 years [18]. However, other groups have reported observations similar to those in this study, albeit from retrospective studies conducted in older children [19-21]. Interestingly, Wickens et al. [22] reported that antibiotic exposure in the first 15 months of infancy was associated with the development of AD (OR 1.83, 95\% CI 1.10-3.05) and rash (OR 1.61, 95\% CI 1.02-2.53) after 15 months, which persisted till 4 years in a birth cohort. This is in agreement with our observation that antibiotic exposure in the first 6 months of life was significantly associated with the development of doctor-diagnosed AD. Kuo et al. [23] suggested that this could be due to the suppression of production of pro-Th 1 cytokines, IL- 6 and TNF- $\alpha$ by antibiotics [24], resulting in a predominant Th2 environment in the infant. Infants are born with Th2-skewed immune responses and infections may skew towards a Th1 response according to the hygiene hypothesis. Antibiotics also disrupt the gut microflora, potentially tipping the immune response balance towards the Th2 predominance associated with allergic diseases [25].

We found that early-onset doctor-diagnosed $\mathrm{AD}$ was associated with allergen sensitization, while late-onset $\mathrm{AD}$ was not. This suggests that allergy may be associated with early AD but not late-onset AD. Associations between early-onset $\mathrm{AD}$ and positive SPT reactivity to aeroallergens are well established $[26,27]$. Our findings are consistent with a defective skin barrier due to AD supporting the dermal route of sensitization to aeroallergens $[28,29]$.

The limitations of our study were that the information was self-reported and there may have been recall and information bias. In addition, the design of the question- naire did not allow us to phenotype into transient and persistent eczema cases. Longer follow-up will be needed to establish the proposed phenotypes. The strengths of this study were the large sample size and the prospective collection of data from the prenatal stage.

In conclusion, we found that early-onset $\mathrm{AD}$ was mainly associated with familial factors, such as maternal allergy, while late-onset $\mathrm{AD}$ was associated with antibiotic treatment or the consumption of probiotics. Both familial and environmental factors as well as antibiotic treatment were associated with the development of AD between the ages of 6 and 12 months. Our study supports the concept that different phenotypes of $\mathrm{AD}$ exist in young children.

\section{Acknowledgements}

This work was supported by the National Medical Research Council, (NMRC)/CSA/022/2010 and National Research Foundation (NRF) 370062-HUJ-NUS (Project 10) and the Translational Clinical Research Flagship Program on Developmental Pathways to Metabolic Disease which is funded by the NRF and administered by the NMRC, Singapore (NMRC/TCR/004-NUS/2008). K.M.G. is supported by the National Institute for Health Research (Southampton Biomedical Research Centre) and the European Union's Seventh Framework Programme (FP7/2007-2013), project EarlyNutrition under grant agreement No. 289346. We acknowledge the contribution of the rest of the GUSTO study group: Pratibha Agarwal, Dennis Bier, Arijit Biswas, Shirong Cai, Jerry Kok Yen Chan, Cornelia Yin Ing Chee, Helen Y.H. Chen, Audrey Chia, Amutha Chinnadurai, Chai Kiat Chng, Mary Foong-Fong Chong, Shang Chee Chong, Mei Chien Chua, Chun Ming Ding, Eric Andrew Finkelstein, Doris Fok, Marielle Fortier, Yam Thiam Daniel Goh, Joshua J. Gooley, Wee Meng Han, Mark Hanson, Christiani Jeyakumar Henry, Joanna D. Holbrook, Chin-Ying Hsu, Hazel Inskip, Jeevesh Kapur, Yung Seng Lee, Birit LeutscherBroekman, Sok Bee Lim, Seong Feei Loh, Yen-Ling Low, Iliana Magiati, Lourdes Mary Daniel, Michael Meaney, Susan Morton, Cheryl Ngo, Krishnamoorthy Niduvaje, Wei Wei Pang, Anqi Qiu, Boon Long Quah, Victor Samuel Rajadurai, Mary Rauff, Jenny L. Richmond, Anne Rifkin-Graboi, Allan Sheppard, Borys Shuter, Leher Singh, Wing Chee So, Walter Stunkel, Lin Lin Su, Kok Hian Tan, Soek Hui Tan, Mya Thway Tint, Rob M. van Dam, Sudhakar K. Venkatesh, Inez Bik Yun Wong, P.C. Wong, Fabian Kok Peng Yap and George Seow Heong Yeo.

\section{Disclosure Statement}

Peter D. Gluckman, Keith M. Godfrey, Lynette Pei-chi Shek and Yap-Seng Chong have received reimbursement for speaking at conferences sponsored by companies selling nutritional products. Peter D. Gluckman, Keith M. Godfrey, and Yap-Seng Chong are part of an academic consortium that has received research funding from Abbot Nutrition, Nestec and Danone. Lynette Peichi Shek has received research funding from Danone. 


\section{References}

1 Asher MI, et al: Worldwide time trends in the prevalence of symptoms of asthma, allergic rhinoconjunctivitis, and eczema in childhood: ISAAC phases one and three repeat multicountry cross-sectional surveys. Lancet 2006;368:733-743.

2 Bjorksten B: Effects of intestinal microflora and the environment on the development of asthma and allergy. Springer Semin Immunopathol 2004;25:257-270.

3 Wang XS, et al: The prevalence of asthma and allergies in Singapore; data from two ISAAC surveys seven years apart. Arch Dis Child 2004;89:423-426.

4 Spergel JM: Atopic march: link to upper airways. Curr Opin Allergy Clin Immunol 2005; 5:17-21.

5 Kay J, et al: The prevalence of childhood atopic eczema in a general population. J Am Acad Dermatol 1994;30:35-39.

6 Tay YK, et al: The prevalence and descriptive epidemiology of atopic dermatitis in Singapore school children. Br J Dermatol 2002;146:101-106.

7 Tan TN, et al: Prevalence of allergy-related symptoms in Singaporean children in the second year of life. Pediatr Allergy Immunol 2005; 16:151-156.

8 Spergel JM, Paller AS: Atopic dermatitis and the atopic march. J Allergy Clin Immunol 2003;112(suppl):S118-S127.

9 Soh SE, et al: The methodology of the GUSTO cohort study: a novel approach in studying pediatric allergy. Asia Pac Allergy 2012;2: 144-148.

10 Soh SE, et al: Cohort profile: growing up in Singapore towards healthy outcomes (GUSTO) birth cohort study. Int J Epidemiol 2014;43:1401-1409.

11 ISAAC Phase One Manual, ed 2. December 1993, pp 10-12. http://isaac.auckland.ac.nz/ story/methods/resources/phaseonemanual. pdf.

12 Kusel MM, et al: Support for 2 variants of eczema. J Allergy Clin Immunol 2005;116: 1067-1072.

13 Harris JM, et al: Environmental associations with eczema in early life. Br J Dermatol 2001; 144:795-802.

14 Belyhun Y, et al: Prevalence and risk factors of wheeze and eczema in 1-year-old children: the Butajira birth cohort, Ethiopia. Clin Exp Allergy 2010;40:619-626.

15 Lee JY, et al: Exposure to gene-environment interactions before 1 year of age may favor the development of atopic dermatitis. Int Arch Allergy Immunol 2012;157:363-371.

16 Risnes KR, et al: Antibiotic exposure by 6 months and asthma and allergy at 6 years: findings in a cohort of 1,401 US children. Am J Epidemiol 2011;173:310-318.

17 Celedon JC, et al: Antibiotic use in the first year of life and asthma in early childhood. Clin Exp Allergy 2004;34:1011-1016.

18 Kummeling I, et al: Early life exposure to antibiotics and the subsequent development of eczema, wheeze, and allergic sensitization in the first 2 years of life: the KOALA birth cohort study. Pediatrics 2007;119:e225-e231.

19 Garcia-Marcos L, et al: Early exposure to paracetamol or to antibiotics and eczema at school age: modification by asthma and rhinoconjunctivitis. Pediatr Allergy Immunol 2010;21:1036-1042.

20 Foliaki S, et al: Antibiotic use in infancy and symptoms of asthma, rhinoconjunctivitis, and eczema in children 6 and 7 years old: International Study of Asthma and Allergies in Childhood Phase III. J Allergy Clin Immunol 2009; 124:982-989.

21 Lee SY, et al: Prevalence of atopy and allergic diseases in Korean children: associations with a farming environment and rural lifestyle. Int Arch Allergy Immunol 2012;158: 168-174.

22 Wickens $\mathrm{K}$, et al: The association of early life exposure to antibiotics and the development of asthma, eczema and atopy in a birth cohort: confounding or causality? Clin Exp Allergy 2008;38:1318-1324.

$23 \mathrm{Kuo} \mathrm{CH}$, et al: Early life exposure to antibiotics and the risk of childhood allergic diseases: an update from the perspective of the hygiene hypothesis. J Microbiol Immunol Infect 2013; 46:320-329.

24 Shalit I, et al: Anti-inflammatory effects of moxifloxacin on IL-8, IL-1beta and TNF-alpha secretion and NFkappaB and MAP-kinase activation in human monocytes stimulated with Aspergillus fumigatus. J Antimicrob Chemother 2006;57:230-235.

25 Oyama N, et al: Antibiotic use during infancy promotes a shift in the $\mathrm{T}(\mathrm{H}) 1 / \mathrm{T}(\mathrm{H}) 2$ balance toward $\mathrm{T}(\mathrm{H}) 2$-dominant immunity in mice. J Allergy Clin Immunol 2001;107:153159

26 Schafer T, et al: Association between severity of atopic eczema and degree of sensitization to aeroallergens in schoolchildren. J Allergy Clin Immunol 1999; 104:1280-1284.

27 Capristo C, Romei I, Boner AL: Environmental prevention in atopic eczema dermatitis syndrome (AEDS) and asthma: avoidance of indoor allergens. Allergy 2004;59(suppl 78): 53-60.

28 Strid J, Strobel S: Skin barrier dysfunction and systemic sensitization to allergens through the skin. Curr Drug Targets Inflamm Allergy 2005;4:531-541.

29 Carlsten C, et al: Atopic dermatitis in a highrisk cohort: natural history, associated allergic outcomes, and risk factors. Ann Allergy Asthma Immunol 2013;110:24-28. 